\title{
Digital quality control of construction work
}

\author{
Jaroslav Synek ${ }^{1, *}$ \\ ${ }^{1}$ CTU Prague, Department of Building Technology, Thakurova 7, 16059 Praha 6, Czechia, \\ and Metrostav, a.s., Koželužská 2450, 18000 Praha 8, Czechia
}

\begin{abstract}
This paper focuses on deploying, using and evaluating the benefits of digital quality control on site - requirements for digital control, digital control principles, digital control tools and their capabilities, outputs from digital quality control, utilization and implementation of digital quality control and its benefits for design, implementation and evaluation in the building's life cycle.
\end{abstract}

\section{Introduction - Need to increase productivity in the construction industry}

Labour productivity lagging compared to other industries is about $20-25 \%$. The problem is generally attributed to insufficient digitization of the construction industry. The reasons are more complex, the primary cause is the marked difference between industrial and construction production. Digitization is only a powerful tool that does not replace engineering inventions and feelings, but it is able to greatly increase productivity of availability and sharing of information and processes. Allows work with up-to-date information and reduces recurring, unproductive activities that occupy a significant portion of working time.

\subsection{The Digitization of Construction and Construction 4.0}

The term Construction 4.0 appears in this context in the Czech Republic. The concept is inspired by the German idea Industry 4.0, but works with different starting points for the construction industry.

\subsection{Characteristics of construction projects}

Characteristic features of construction projects are the long preparation and realization period (months to years), and the associated amount of changes in the technical solution and over time, great diversity, many suppliers. The result is complex project management. [1]. The most problems are the processing of changes that are slow and inaccurate, inefficient. Digitization rationalizes procedures, refines and enables them to be reviewed.

*Corresponding author: jaroslav.synek@,fsv.cvut.cz 


\section{$1.33 \mathrm{C}$ - prerequisites for digital efficiency}

Shortcut $3 \mathrm{C}$ summarizes the necessary requirements and prerequisites for successful digital technology, i.e. professional competence, co-operation in project operations, and coordination of participants' activities aimed at achieving project goals.

\subsection{Quality and work with information, communication}

One of the major building quality problems is the cyclical loss of information during the life cycle processes of the building, especially when passing information between subjects, entities, small subsequent availability for further use and archiving. The loss of information stems, among other things, from the fact that the author, processors of information often differ in the various phases of the project. Some subjects work separately for the client, others for the contractor. The level of cooperation and communication between them is very limited. Therefore, cooperation and communication need to be improved and accelerated - to make it more effective. Effective communication is a prerequisite for effective project management. Insufficient level of communication in the project or its unreadable content are often the cause of inefficient and error-related activities, delays, most often in the field of changes, alternatives and their approval. Documenting communication documents operations with data over the project.

\subsection{Common Data Environment (CDE)}

The communication and storage environment (a server accessible to project participants) allows for the necessary communication, data co-operation, document management, acceleration of processes and their transfer to implementation. The shared documented communication, co-operation and competence of project participants leads to a reduction in the usual loss of information.

\section{Quality and accuracy - always up-to-date information}

Managing changes in the project is another cause of losses and mistakes during preparation and implementation, like inadequate or ineffective administration, the unavailability of valid supporting documents. Digitization makes it possible to speed up and refine the administration of a single data environment (CDE). Its use limits inaccuracies, accuracy and speed of processes is increasing.

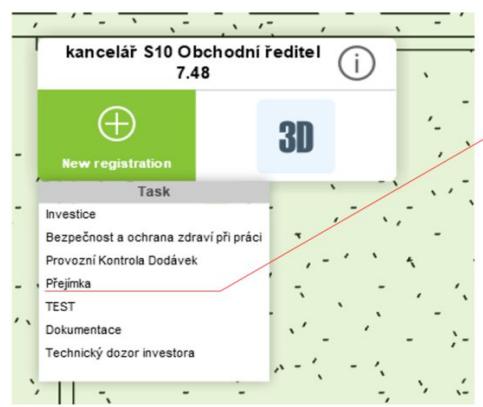

Fig. 1. The example of creating a checklist

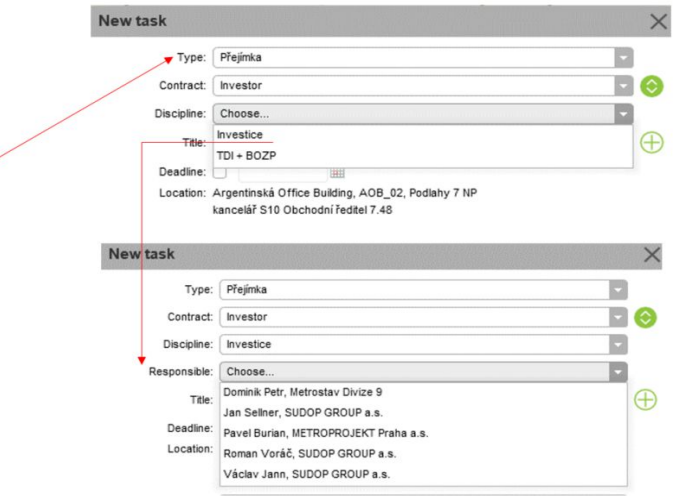




\subsection{Higher efficiency of work - lower costs}

Digitization streamlines, streamlines and accelerates processes, reduces costs by reducing losses and inaccuracies.
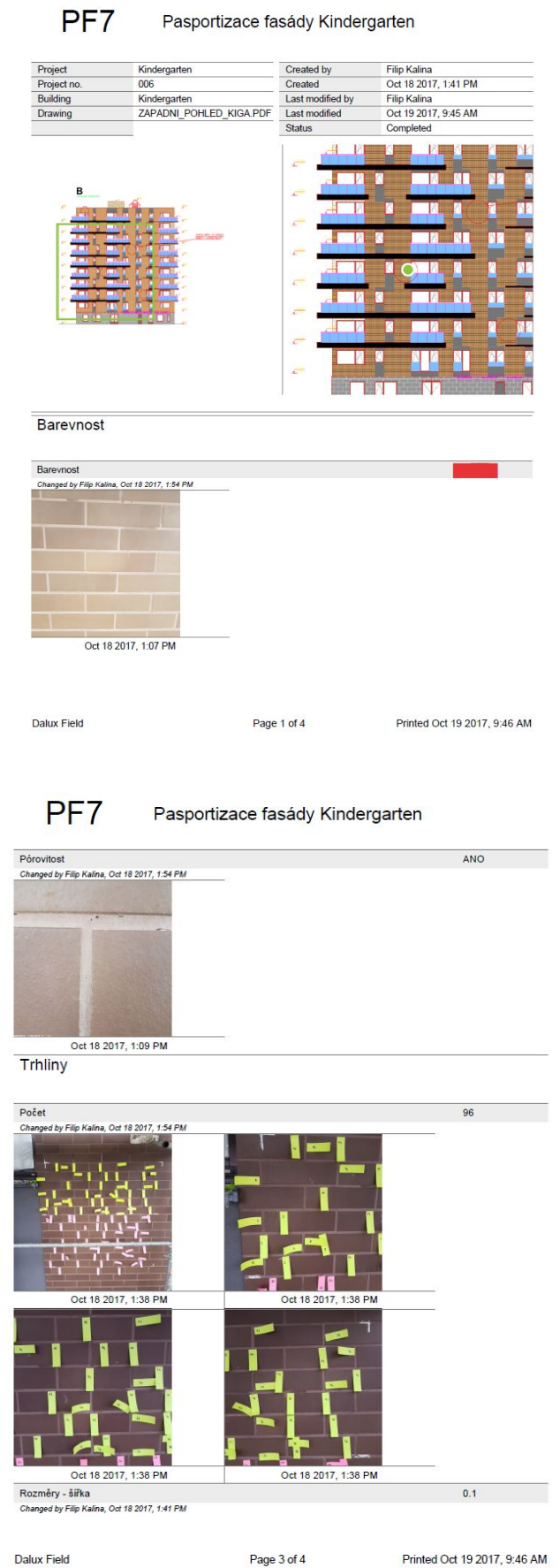

Fig. 2. The example of digital control output 


\subsection{Feedback}

Digital technologies, their processes and outputs allow to evaluate effectiveness in relation to project objectives as a basis for increasing their efficiency, allow for the search for risk processes, to detect and eliminate their causes as a process of improving process management and quality.

\subsection{Quality - client expectations}

Product quality has long been among the highly-respected aspects of client selection. That is why it is necessary for the project participants to focus on the quality of processes in the preparation and implementation of the product as a condition of final client quality.

\section{Quality management goals and requirements identification}

Quality management is designed to ensure product compliance with client requirements. Therefore, it is necessary to achieve client compliance with the requirements for preparation and implementation.

Generic client quality goals usually include long-term reliability, persistent downtime, consistency of expected and actual user requirements, and their parameters. Manufacturers of the entire product and its parts must be able to identify individual client requirements and their parameters and transfer them to the implementation procedures. It is equally important to identify the processes and procedures of both the requirements (parameters) and the procedures to keep the parameters for a set time. [2, 3]

\subsection{Current state of quality management and its shortcomings}

Current quality management practices are based on the CSN EN ISO 9000 standards. These are primarily focused on maintaining and administering quality management procedures, less on improving and improving the quality of implementation. The ISO 9000 quality management system has little use of feedback to improve process control, although it works with quality control outputs and non-conforming products (operations). To improve the quality, it is necessary to work efficiently with information from the preparatory, implementation and operational processes, to use the feedback, to apply the results of the quality evaluation. [2] 


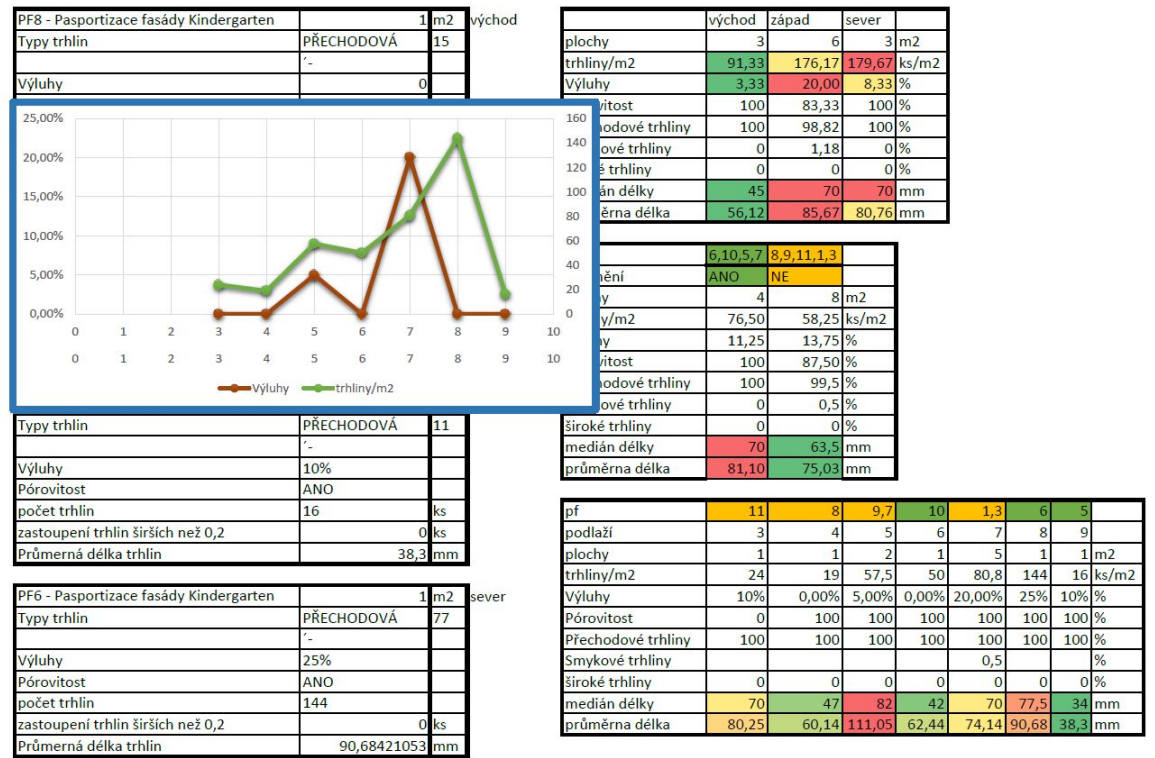

Fig. 3. Evaluation of control results

\subsection{Using Quality Score Results}

Feedback information is generated by quality checks in preparation, implementation and use. The maximum can be obtained during the implementation and during the use in the warranty period, when the product is subject to increased attention and the checks are carried out either according to Inspection and Examination Plans (realization), or according to the principles of acceptance and monitoring of the client. Less intense quality controls take place in the design phase of production, design, design documentation, although their results are critical to quality design. [3]

\subsection{Output Quality Assessment Requirements}

The information obtained must be classified and evaluated in relation to the client's requirements and contractor's procedures, to archive them and to work with them in the long term, especially for the prevention of defects, removal of their causes. The digitization of processes, inputs and outputs is a way to share, accelerate and refine processes of control, evaluation of results and their use for new projects. The results and their processing and evaluation give feedback to all participants in building projects, while ensuring their availability for long-term use.

\section{Reasons for digitization of control and necessary assumptions}

Current undigested control procedures are laborious, inaccurate, and usually unavailable for use after the end of the project. Digitization changes processes, outputs, and uses of quality control results. It makes them more efficient for other processes in the life cycle of the building, even in the preparation of other projects. 
Quality control generally assumes unambiguously defined requirements at each stage of the project (design, implementation, approval processes and operation). The digitization of control refines and accelerates activities, brings added value in the possibility of using the results on a lasting basis by sharing them, but requires the necessary qualification prerequisites for the participants in the project.

\subsection{Principles and needs of digital control}

Digital control processes use digital material (technical, control, control) for processing, records, outputs and distribution. The digital form allows online communication over records, co-operation and coordination of subsequent operations (defect removal).

The shortcomings of the current digitization procedures eliminate, allowing processes and operations to perform once, usually on site, evaluation and processing of the record. The result of the inspection, the assessment is recorded on the spot in the approved protocol, which is also the output of the inspection. The output is distributed on-line, upon completion, according to the distribution list of persons charged with contract-based control. This greatly reduces the need for working time for control and communication.

\subsection{Digital backgrounds and technologies}

Digital data (e.g. project documentation), as well as digitized documents (statements, opinions, decisions, technical reports, technical sheets, etc.) use only pre-arranged data formats for the operations under consideration. Even the most common formats (PDF) for mobile devices (tablet, smartphone) can usually be used.

Required control applications installed on mobile devices communicate in a common data environment (CDE). Records work (slides, measurements, descriptions of checked items), edits logs and defect removal, defines results, usually allows full-text search. CDE permits the storage, access and other operations of competent persons in the project team of both the investor and the contractor, often not only for the duration of the project. This data is therefore useful for feedback over the long term.

\subsection{Long-term usability of results and statistical evaluation of results}

The resulting information (protocols, evaluations, outputs) is automatically provided with appropriate metadata that allows the systematic use of shared data over the long term of the life cycle of a building.

The content of the controls sets out a contract (agreement) between the client and the contractor on the content, format and outputs of the control and test plan. Similarly, its digitized version is ready.

The check and test plan usually records the place, time, frequency, conditions, test procedures with reference to the prescribed tests (according to the technical standards or, as the case may be, according to the project participants' agreement), performance requirements, test results and statement (pass / fail).

The digital form of information allows fast search and orientation in documents, using the right data format allows you to apply the necessary calculations, operations and procedures for processing and displaying the results. Unification of data formats makes it easy to use computational procedures for evaluation and comparison. 


\section{Use of digital techniques and operational benefits for design and implementation}

The key benefit of digitization is the significant acceleration and refinement of control activities not only on the site, the exclusion of non-systemic hand transfers of information into control protocols and the addition of images and records, replacement of problematic communications.

\subsection{High efficiency - shortening the processing time}

Digital form and processing procedures allow standardization of procedures and protocols for use throughout the project. These procedures allow you to process a copy directly at the control site, incl. processing of attachments, pictures and records of defects and deficiencies directly to the protocol, incl. evaluation - control results. Direct processing of the protocol at the site of control significantly reduces the working time of workers. High efficiency enables quality control to focus on truly critical operations.

Metadata outputs allow any time to identify processes and results by location, time, position, and subject matter, detected defects, selected procedures, and so on. They allow quick search of available files for the cumulative evaluation of certain types of constructions, procedures, objects, and results in available information files. They can be used to systematically assess the partial risks of projects and operations. Using the available storage limits the usual recurring activity of using the information obtained.be numbered with the number on the right-hand side.

\subsection{Unambiguity, accuracy and quality}

A big problem with the current quality of the buildings is the ambiguity of the documentation. This is widespread, unclear and, in spite of the requirements of legal and technical regulations, is usually poorly coordinated. This is due to the complex processes of creating documentation as a collective work that changes as the knowledge of the project issues develops. These procedures are similar to the implementation changes that the contractor must make during implementation and process the actual execution documentation.

Pre-approved forms and checklists allow for high accuracy, speed and record-keeping, are unambiguous, metadata defined, records archived, available for further use. Such outputs are inconsistent with current practice in terms of content and scope, usability incompatible with current practice, non-systematic manual filing of checklists.

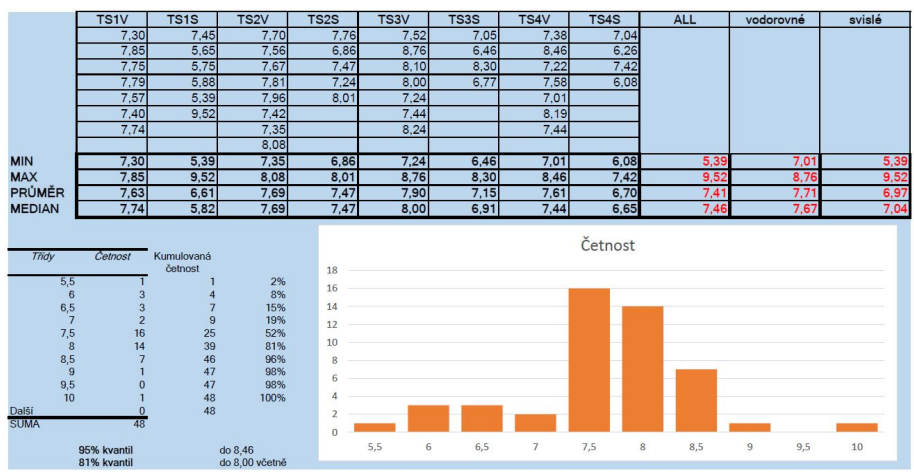

Fig. 4. Statistical evaluation of control results. 


\section{Use of digital techniques}

The use of digital control tools with ready-made control forms for use in both internal and external work control, greatly increases the productivity of site control activities.

\subsection{Streamlining - Acceleration, Refinement, One Activity}

Limitation of routine non-systemic activities, reduction of finishing errors, unambiguous identification (subject, place and time of inspection, used control methods and procedures, images and results, communication and responsibility, archiving, etc.) is the key of higher efficiency. Activities are not duplicated.

Time savings, refinements and high efficiency of control activities, and limitations of non-system procedures clearly favour digital practices. Control workers who have tried digitized procedures for checking, processing, and sharing documents are no longer returning to manual processing.

Digital building technologies are not yet widely used, but each of their partial use significantly improves management efficiency. Using digital technologies brings increased accuracy and processing speed, accessible to all project participants, in conventional building management processes. This allows gradual transition of project management to full digitization.

\section{Conclusion}

Proven benefits include unification, operational processing and optimization of outputs, standardization of operational procedures, acceleration, higher quality and less error of information. Information is accurate, unambiguous, up-to-date, available, and provides feedback to improve production. Digital information allows connection to other documentation and digital processes.

The gradual introduction of digital operations is a way of extending the knowledge of project teams, leading to more efficient production, higher quality and higher productivity of the construction industry. The method of gradually introducing digital processes into building management brings a demonstrable increase in productivity. It is a good way to gradually introduce and expand digital processes across the building industry.

\section{References}

1. A. P. C. Chan, Architectural Science Review, 39(2), 107 - 112 (1996)

2. T. Benitto, J. Arunima, International Journal of Civil Engineering and Technology, 8(4), 792-800 (2017) Available on https://www.researchgate.net/publication/320149474_Quality_Management_System a t_Construction_Project_A_Questionnaire Survey

3. P. P. Mane, J. R. Patil, Int. Journal of Engineering Research and Applications 5(3), 126-130 (2015) 